\title{
Assessment of Epidemiology Capacity in State Health Departments, 2004-2009
}

Matthew L. Boulton, MD, $\mathrm{MPH}^{\mathrm{a}, \mathrm{b}}$

James HadLer, MD, MPH

ANGELA J. BEGK, MPH, CHES

Lisa FerLand, MPH

Maureen LichtVeld, MD, MPH

\section{SYNOPSIS}

Objectives. To assess the number of epidemiologists and epidemiology capacity nationally, the Council of State and Territorial Epidemiologists surveyed state health departments in 2004, 2006, and 2009. This article summarizes findings of the 2009 assessment and analyzes five-year (2004-2009) trends in the epidemiology workforce.

Methods. Online surveys collected information from all 50 states and the District of Columbia about the number of epidemiologists employed, their training and education, program and technologic capacity, organizational structure, and funding sources. State epidemiologists were the key informants; 1,544 epidemiologists provided individual-level information.

Results. The number of epidemiologists in state health departments decreased approximately $12 \%$ from 2004 to 2009. Two-thirds or more states reported less than substantial ( $<50 \%$ of optimum) surveillance and epidemiology capacity in five of nine program areas. Capacity has diminished since 2006 for three of four epidemiology-related Essential Services of Public Health (ESPHs). Fewer than half of all states reported using surveillance technologies such as Webbased provider reporting systems. State health departments need $68 \%$ more epidemiologists to reach optimal capacity in all program areas; smaller states ( $<5$ million population) have higher epidemiologist-to-population ratios than more populous states.

Conclusions. Epidemiology capacity in state health departments is suboptimal and has decreased, as assessed by states' ability to carry out the ESPHs, by their ability to use newer surveillance technologies, and by the number of epidemiologists employed. Federal emergency preparedness funding, which supported more than $20 \%$ of state-based epidemiologists in 2006, has decreased. The 2009 Epidemiology Capacity Assessment demonstrates the negative impact of this decrease on states' epidemiology capacity.

${ }^{a}$ Center of Excellence for Public Health Workforce Studies, University of Michigan School of Public Health, Ann Arbor, MI

${ }^{b}$ University of Michigan Medical School, Ann Arbor, MI

'Council of State and Territorial Epidemiologists, Atlanta, GA

dDepartment of Environmental Health Sciences, Tulane University School of Public Health and Tropical Medicine, New Orleans, LA

Address correspondence to: Matthew L. Boulton, MD, MPH, Center of Excellence for Public Health Workforce Studies, University of Michigan School of Public Health, 1415 Washington Heights, Ann Arbor, MI 48109; tel. 734-764-6478; fax 734-764-9293; e-mail <mboulton@umich.edu>.

(C2011 Association of Schools of Public Health 
A number of studies have predicted dramatic shortages of public health workers, ${ }^{1-3}$ prompting calls from professional groups for increases in federal investment in the public health workforce. ${ }^{3-5}$ The Council of State Governments and the National Association of State Personnel Executives' 2002 workforce survey of state agencies concluded that state governments could lose more than $30 \%$ of their workforce to retirement and alternative careers within the next five years; health agencies were predicted to suffer the greatest personnel losses. In 2003, a survey by the Association of State and Territorial Health Officials (ASTHO) documented a rapidly aging state public health workforce, a high proportion of workers eligible for retirement, chronic vacancy rates for state health department jobs, and high turnover rates in people who are already employed. ${ }^{1}$

Although these issues impact the entire public health workforce, they may disproportionately affect the epidemiology workforce, which constitutes only $1 \%$ of public health workers in state and local health departments, according to a 2007 Bureau of Labor and Statistics assessment. ${ }^{6,7}$ ASTHO reported that $41 \%$ of states responding to a state public health employee survey had a shortage of epidemiologists, second only to nurses. ${ }^{1}$ These findings were supported by its 2007 public health workforce survey characterizing epidemiologists as moderately to very affected by workforce shortages. ${ }^{2}$ A 2008 survey by the National Association of County and City Health Officials found that only $23 \%$ of the nation's local health departments employed an epidemiologist, and the number of epidemiologists had decreased by almost $8 \%$ since $2005 .{ }^{8}$ Although small, the epidemiology workforce constitutes the scientific core of public health, and epidemiologists are considered essential to monitoring, detecting, controlling, and preventing population health hazards, such as pandemic influenza.

The Council of State and Territorial Epidemiologists (CSTE), the professional association for epidemiologists working in states, local health agencies, and territories, conducted four national Epidemiology Capacity Assessments (ECAs) in 2001, 2004, 2006, and 2009 to evaluate epidemiology capacity in state health departments and monitor progress toward achieving the Healthy People 2010 Objective 23-14: "increase the proportion of Tribal, state and local public health agencies that provide or assure comprehensive epidemiology services to support essential public health services [so] they can quickly detect, investigate, and respond to diseases to prevent unnecessary transmission." ${ }^{9} \mathrm{An}$ earlier analysis of six-year trends (2001-2006) in states' epidemiology capacity based on CSTE surveys showed an overall increase in epidemiologists and epidemiol- ogy capacity, although at levels substantially below full capacity for most program areas. ${ }^{5,10-12}$ That study found a strong statistical correlation between population size and the number of epidemiologists, which was used as a basis for recommending a ratio of one epidemiologist per 100,000 population as an approximate gauge of sufficient epidemiology capacity. ${ }^{5}$

The survey methodology and analysis used for the 2009 ECA differed from that of previous epidemiology capacity assessments in several important ways. Individual-level data on epidemiologists' training and educational background were collected for the first time, which also permitted estimates of epidemiology program capacity based on individual (vs. organizational) reporting; a new module on states' laboratory and disease reporting technical capacity was added and analyzed as part of the current assessment; substance abuse program capacity was newly assessed; and enumeration data were stratified by state size for analysis. This article summarizes the findings of the 2009 ECA, evaluates five-year trends (i.e., 2004-2009) in state health departments' epidemiology capacity, and elaborates on the recommended ratio of epidemiologists to population served.

\section{METHODS}

In April 2009, we distributed the ECA in electronic and paper formats to all 50 states, the District of Columbia (DC), and eight U.S. territories after pilot testing it in three states. ${ }^{13}$ CSTE staff provided help sessions and technical assistance to epidemiologists completing the survey during the data-collection period. State Epidemiologists or their delegate(s) were the designated key informants for the Core Assessment. The State Epidemiologist was also asked to send electronic worksheets to all employees working in the state health department who met the definition of epidemiologist to provide individual-level data. We defined "epidemiologist" as in previous ECAs, using Last's 2001 edition of A Dictionary of Epidemiology. 13,14

CSTE e-mailed each state's reported enumeration information to the State Epidemiologist for review and verification. CSTE staff phoned State Epidemiologists who did not verify the enumeration responses and in whose states the number of epidemiologists reported in 2006 and 2009 varied by more than $10 \%$; they were asked only to verify the total number of epidemiologists reported. Data collection was completed by June 30, 2009.

The Core Assessment, completed by the State Epidemiologist, comprised three modules. Module 1 collected summary department-level information related

Public Health Reports / January-February 2011 / Volume 126 
to overall funding sources, the State Epidemiologist position, the epidemiology-related Essential Services of Public Health (ESPHs) ${ }^{15}$ program area capacity, and worker salaries. The 2009 ECA addressed nine program areas: bioterrorism/emergency response (BT/ER), chronic diseases, environmental health, infectious diseases, injury, maternal and child health $(\mathrm{MCH})$, occupational health, oral health, and substance abuse. It also addressed four ESPHs: (1) monitor health status to identify and solve community health problems (ESPH $1)$; (2) diagnose and investigate health problems and health hazards in the community (ESPH 2); (3) evaluate the effectiveness, accessibility, and quality of personal and population-based health services (ESPH 9); and (4) research for new insights and innovative solutions to health problems (ESPH 10). Respondents assessed capacity on a six-category scale based on the proportion of activity, knowledge, or resources met for each ESPH and epidemiology program area. Responses were collapsed into two categories: none-to-partial $(0 \%$ to $<50 \%$ ) or substantial-to-full ( $50 \%$ to $100 \%$ ).

Module 2 focused on data collection related to recruitment and retention of epidemiologists and organizational research productivity. Module 2 data are not presented in this article and will be analyzed in a forthcoming article. Module 3 focused on technical epidemiology capacity, including use of electronic laboratory reporting (ELR), Web-based provider reporting, disease databases meeting specific Centers for Disease Control and Prevention (CDC) standards, geo-coding, and outbreak management systems.

Reponses from individual epidemiologists employed by the state health department were used to determine the total number of epidemiologists by program area, the number of epidemiologists supported with federal funds, retirement plans, and levels of education and epidemiology training.

We analyzed data using $\mathrm{SAS}^{\oplus}$ version 8.0, ${ }^{16} \mathrm{SPSS}^{\oplus}$ version 17.0, ${ }^{17}$ and Microsoft ${ }^{\oplus}$ Excel software. We stratified enumeration data by state population for analysis, and we calculated trend data for number of epidemiologists by program area from State Epidemiologists' responses for the 2004 ECA and individual-level data for 2009. Because the 2009 ECA captured these demographic data for only $70 \%$ of epidemiologists, compared with $100 \%$ in the two previous assessments, we compared states for which the number of epidemiologists enumerated based on individual-level responses varied by $\leq 15 \%$ from the number of epidemiologists reported by the State Epidemiologist $(n=28)$ vs. those states where this variation was $>15 \%$. This dichotomization allowed for analysis of variance (ANOVA) testing for two groups of near-equal size.
CSTE did not seek Institutional Review Board (IRB) approval for this study because the data were derived from the de-identified national survey, which does not capture identifier data at the individual level. Such surveys are traditionally exempt from IRB review. Independent consultation with the IRB at the Michigan Department of Community Health confirmed that previous ECA studies would have been eligible for exemption 46.101(b) (2)..$^{5}$

\section{RESULTS}

All 50 state health departments and DC responded to the survey. We excluded U.S. territories from analysis because only two of eight (25\%) territories responded. Denominators for responses varied because not all states answered all questions and some responses used department-level vs. aggregated individual-level data.

\section{Findings of the 2009 ECA}

Core assessment. The 50 states and DC reported employing or contracting a total of 2,193 epidemiologists in 2009-equivalent to 0.72 epidemiologists per 100,000 population (median by state: 0.77 ; range: $0.19-4.05) .{ }^{12,13}$ States estimated needing approximately 1,490 additional epidemiologists to reach full capacity in all program areas, a $68 \%$ increase to 1.21 epidemiologists per 100,000 population. Smaller states (population $<5$ million, $n=30$ ) had a mean of 1.41 epidemiologists per 100,000 population (range: $0.39-4.05$ ) and reported needing a mean of 2.5 epidemiologists per 100,000 population to reach optimal capacity (range: 0.43-6.39). Larger states (population $\geq 5$ million, $n=21$ ) had a mean of 0.67 epidemiologists per 100,000 population (range: $0.19-1.91$ ) and reported needing a mean of one epidemiologist per 100,000 population for optimal capacity (range: 0.32-3.68) (Figure 1). Twenty-one (41\%) states (18 [60\%] of which were small) met or exceeded the recommended epidemiologist-to-population ratio of one per 100,000 population. ${ }^{5}$ Chi-square testing confirmed that meeting or exceeding this ratio varied by state population $(p=0.001)$. Furthermore, logistic regression showed that small states had 2.2 greater odds than large states of meeting or exceeding this ratio $(p=0.002)$.

Estimated capacity in specific program areas and ability to carry out the four epidemiology-related ESPHs varied substantially among states. Reporting of substantial-to-full $(>50 \%)$ capacity of states was $65 \%$ for ESPH 1, 63\% for ESPH 2, 14\% for ESPH 9, and $18 \%$ for ESPH 10. Most states reported substantialto-full capacity for infectious diseases $(92 \%)$, BT/ER $(72 \%), \mathrm{MCH}(55 \%)$, and chronic diseases $(53 \%)$. 
Figure 1. Mean current and optimal number of epidemiologists, by state size: 2009 epidemiology capacity assessment

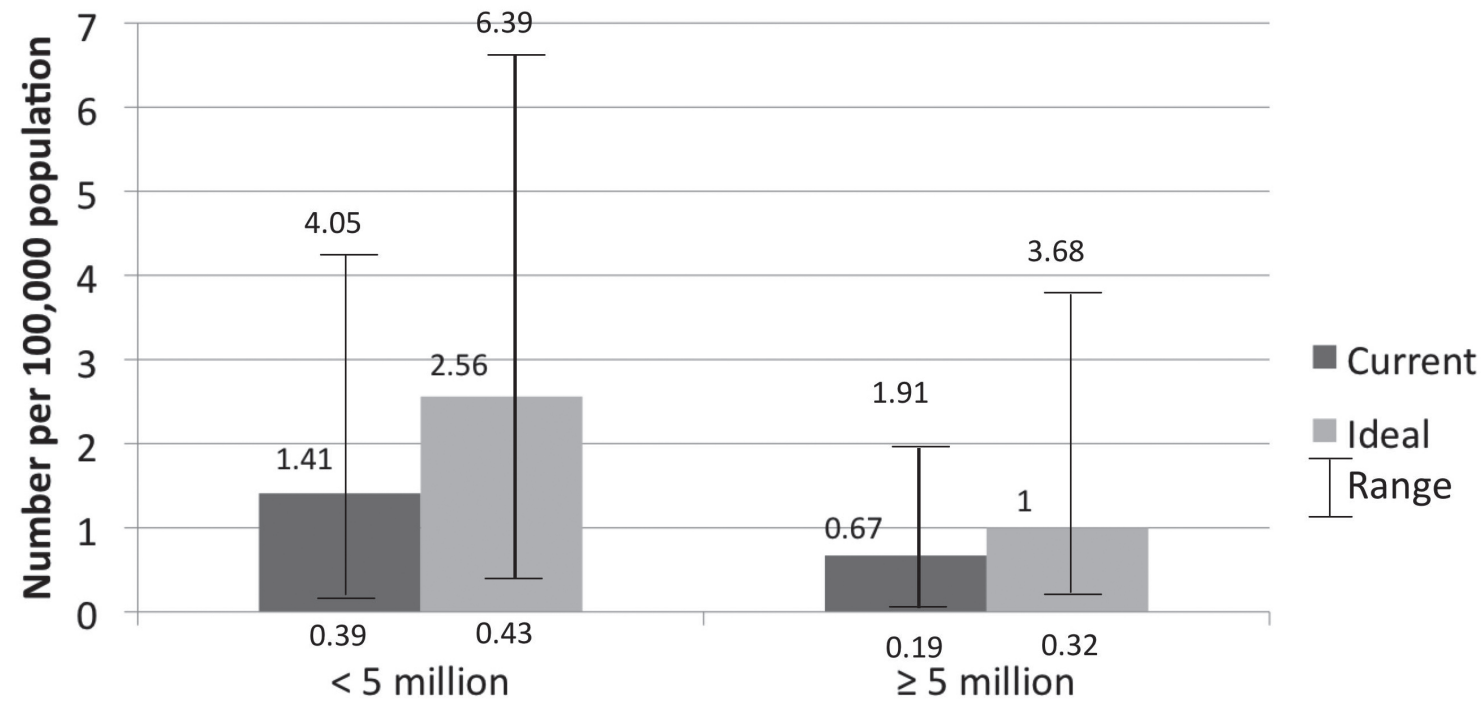

State size

Fewer states reported substantial-to-full capacity for environmental health (38\%), injury (34\%), occupational health $(18 \%)$, substance abuse $(12 \%)$, and oral health $(6 \%)$.

Respondents reported on technical epidemiology capacity of the state health department. More than half $(n=27,53 \%)$ of health departments reported fully functional automated ELR, 10 (37\%) of which had expanded the number of conditions reportable through the ELR system. Fewer than half of the responding states $(n=21,41 \%)$ had a Web-based provider disease-reporting system for completing a case report form online with automatic data entry into a database without reentry. Of the 30 states without a Web-based reporting system, $23(77 \%)$ could not specify when system implementation might occur.

Nearly all $(n=46,90 \%)$ states input reports into a National Electronic Disease Surveillance Systemcompliant database; however, only 12 (24\%) routinely use automated cluster-detection software on reportable diseases and laboratory case report data. Twenty $(39 \%)$ states routinely geo-code all births, 21 (41\%) states geo-code all deaths, and 15 (29\%) states report case data from reportable diseases and laboratory findings. Most $(n=30,59 \%)$ states reported actively working to make electronic medical records useful for public health purposes. Nearly one-third $(n=16,31 \%)$ use an outbreak management system supporting the investigation, response, and containment of outbreaks by collecting and analyzing data.

Individual-level assessment. We analyzed individual-level data from 1,544/2,193 (70\%) epidemiologists, 1,478 of whom were employed full-time. The combined effort of the 1,544 epidemiologists equaled 709 (46\%) fulltime equivalent (FTE) workers in infectious diseases; $178(12 \%)$ FTE workers in chronic diseases; 165 (11\%) FTE workers in BT/ER; 147 (10\%) FTE workers in MCH; 125 (8\%) FTE workers in environmental health; 43 (3\%) FTE workers in injury; 24 (2\%) FTE workers in occupational health; 10 (1\%) FTE workers in substance abuse; and seven $(<1 \%)$ FTE workers in oral health. States reported $136(9 \%)$ FTE workers in other program areas. Most (66\%) respondents had worked at least five years as an epidemiologist. A total of $256(17 \%)$ respondents reported planning to retire or change careers out of epidemiology in the next five years.

Individual-level data on educational background revealed that most epidemiologists $(n=836,54 \%)$ had a Master of Public Health (MPH) (not necessarily in epidemiology) or other master's degree as the highest degree obtained. A total of 262 (17\%) had a Doctor of Philosophy (PhD) or Doctor of Public Health (DrPH) degree, 184 (12\%) had a bachelor's degree, and 167 (11\%) held a Doctor of Medicine (MD) or Doctor of Osteopathic Medicine degree. Fewer epidemiologists 
had a Doctor of Veterinary Medicine degree $(n=50$, $3 \%$ ), associate degree or no post-high school degree $(n=22,1 \%)$, Registered Nurse or other nursing degree $(n=18,1 \%)$, or Doctor of Dental Surgery degree $(n=5$, $<1 \%$ ). Individual-level data specifically on epidemiology education showed that more than half of the epidemiologists had an advanced degree in epidemiology, including an MPH or other master's degree $(n=586$, $38 \%$ ); professional background (e.g., MD) with a dual degree in epidemiology $(n=164,11 \%)$; or $\mathrm{PhD}$, DrPH, or other doctoral degree $(n=121,8 \%)$. Fourteen $(1 \%)$ epidemiologists held a bachelor's degree in epidemiology. Among those without epidemiology degrees, 103 (7\%) epidemiologists had completed formal certificate-level training in epidemiology (e.g., Epidemic Intelligence Service) while others had some coursework $(n=349,23 \%)$ or on-the-job training $(n=177,11 \%)$ as their only epidemiology training. Thirty (2\%) epidemiologists had no formal training of any type in epidemiology (Table).

\section{4-2009: trends in the epidemiology workforce}

All 50 states and DC completed the 2004, 2006, and 2009 ECAs. In 2009, the 51 State Epidemiologists reported 2,193 epidemiologists working in state health departments, a $12 \%$ decrease from the 2,498 reported in 2004 and a $10 \%$ decrease from the 2,436 reported in 2006 (Figure 2).

ANOVA tests comparing the subgroup of 28 states to the remaining 23 states showed no significant differences for all program areas in 2004 and 2009 except for chronic disease programs in 2009, which was significant $(p=0.007)$ (data not shown). Between 2004 and 2009, the number of epidemiologists working in all program areas decreased, with the exception of infectious diseases. Decreases occurred in chronic diseases, from 186 to $138(-26 \%)$; BT/ER, from 157 to $109(-30 \%)$; environmental health, from 143 to 98 $(-31 \%)$; $\mathrm{MCH}$, from 119 to $82(-31 \%)$; injury, from 41 to $29(-29 \%)$; occupational health, from 27 to 17 $(-37 \%)$; and oral health, from 14 to four $(-71 \%)$. The number of epidemiologists working in infectious diseases increased from 426 to $468(+10 \%)$.

The proportion of epidemiologists with a degree, training, or coursework in epidemiology increased with each ECA when those with unknown training level were excluded. In 2004, $137(71 \%)$ of 1,934 epidemiologists reported having a degree, training, or coursework in epidemiology; training was unknown for 564 epidemiologists. In 2006, 1,908 (80\%) of 2,372 epidemiologists reported training in epidemiology, and training was unknown for 84 epidemiologists. In $2009,1,337(87 \%)$ of 1,544 epidemiologists reported training in epidemiology; training was unknown for 790 epidemiologists.

The proportion of states reporting substantial-tofull capacity varied for each of the program areas, with four areas-injury $(+14 \%)$, $\mathrm{MCH}(+10 \%)$, environmental health $(+9 \%)$, and occupational health $(+8 \%)$ - showing increasing capacity since 2004; BT showing decreasing capacity $(-10 \%)$; and infectious diseases $(92 \%$ in 2004, $96 \%$ in 2006 , and $92 \%$ in 2009), chronic diseases (51\% in 2004, $64 \%$ in 2006 , and $53 \%$ in 2009), and oral health $(8 \%$ in $2004,14 \%$ in 2006, and 6\% in 2009) fluctuating in capacity level (Figure 3). State health departments' capacity to carry out the four epidemiology-related ESPHs varied from 2004 to 2009. The percentage of respondents reporting substantial-to-full capacity in ESPH 1 decreased to $65 \%$ in 2009 , after increasing to $80 \%$ in 2006 . Capacity in ESPH 2 decreased from $67 \%$ in 2006 to $63 \%$ in 2009 , which was still higher than the $57 \%$ reported in 2004. Capacity for ESPH 9 decreased to $14 \%$ in 2009 after increasing in 2006 to 39\%. Capacity in ESPH 10 increased slightly to $18 \%$ in 2009 compared with $16 \%$ in 2006 and $12 \%$ in 2004 (Figure 3).

States received a mean of $75 \%$ of their epidemiology program funding from federal sources and 23\% from state funds in the 2009 ECA. Federal funding has increased by $8 \%$ during the past three ECAs $(67 \%$ in 2004 and $71 \%$ in 2006), while state funding has remained level (22\% in 2004 and 23\% in 2006).

\section{DISCUSSION}

The 2009 ECA is the fourth in a series of national assessments of state health department epidemiology capacity conducted during 2001-2009 by CSTE. Regularly and systematically characterizing the public health workforce is essential to improving our ability to monitor the impact of investments and advocate for additional resources; identify gaps in the workforce pipeline; inform recruitment, retention, competency compliance, and credentialing efforts; and ultimately enable us to better understand the links between workforce infrastructure and health outcomes. ${ }^{18}$

The reported number of epidemiologists in state health departments decreased substantially in 2009 relative to that reported three years earlier and was accompanied by corresponding declines in overall functional epidemiology capacity. Prior studies on epidemiology capacity have demonstrated a direct relation between increases in federal preparedness funding and growth in the number of epidemiologists and program capacity. Consequently, the decline in epidemiology capacity is predictable given that federal 


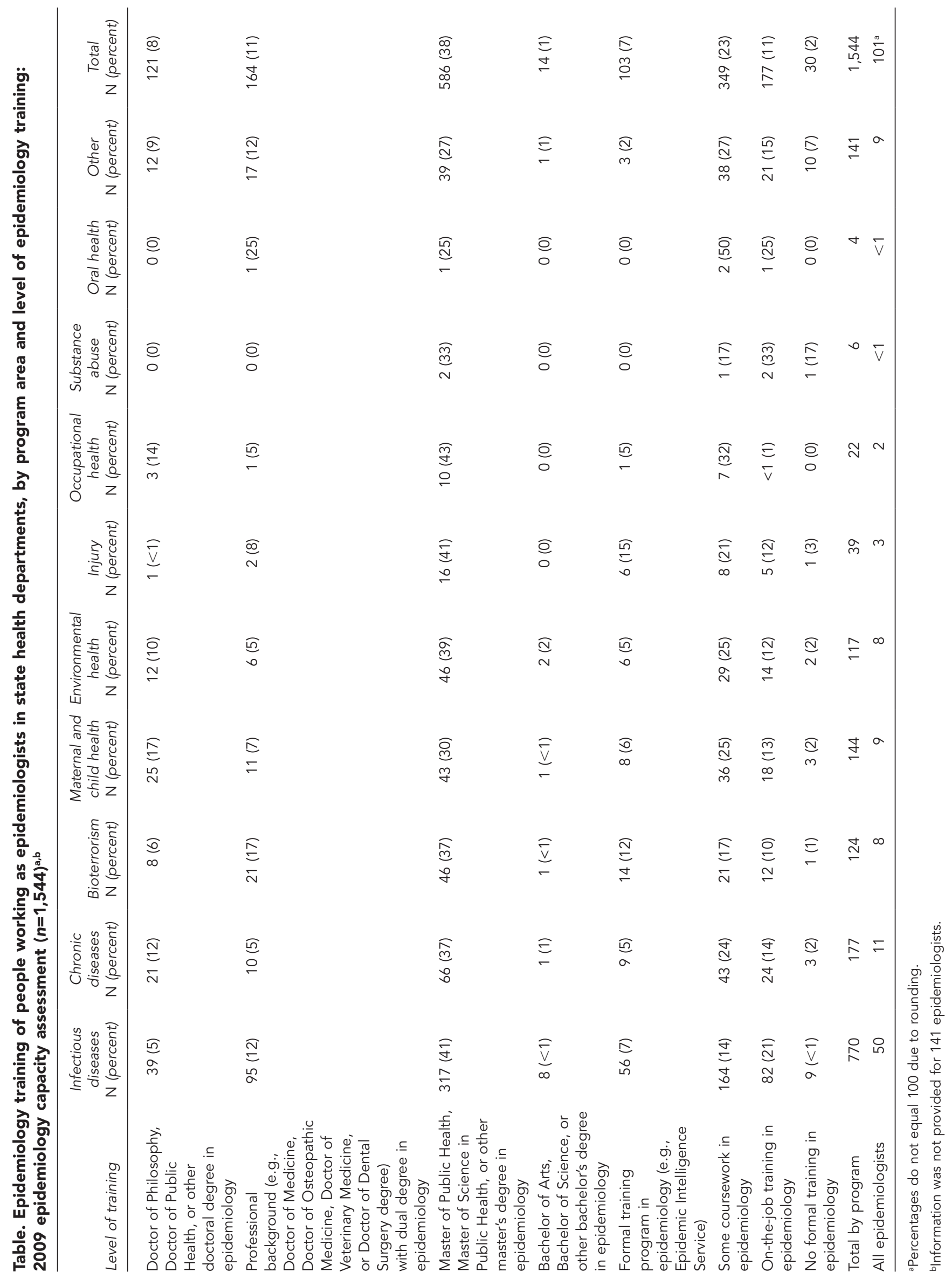

Public Health Reports / January-February 2011 / Volume 126 
Figure 2. Number of state health department epidemiologists and level of training, by survey year: epidemiology capacity assessments, 2004, 2006, and 2009

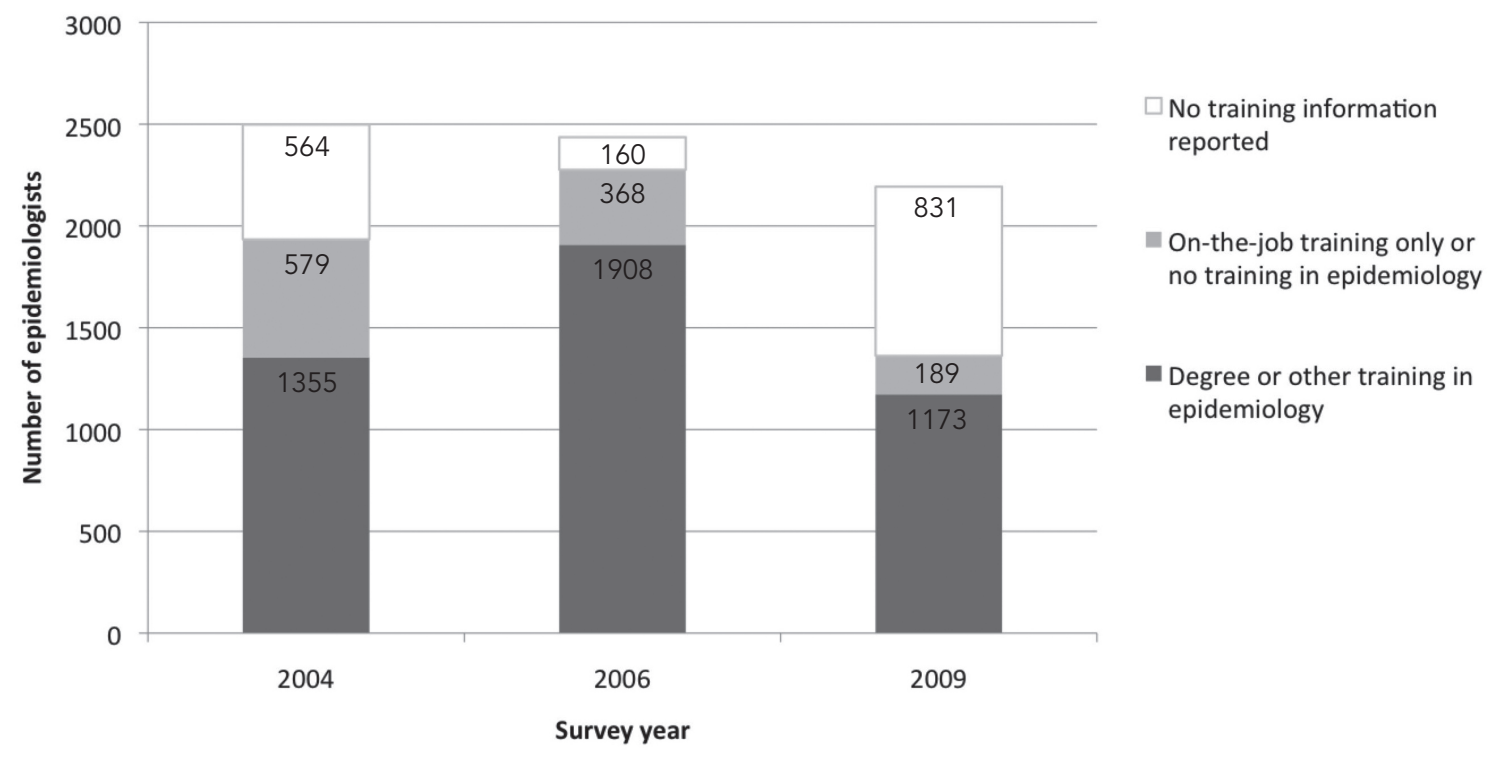

preparedness funding to states is decreasing, worsened by an economic downturn resulting in cuts to state public health budgets. Epidemiology programs and personnel appear vulnerable to loss of federal funding, and unless further decreases in funding are avoided, core epidemiology capacity in state health departments could be imperiled.

Consistent with these losses, State Epidemiologists reported needing $68 \%$ more epidemiologists to reach optimal capacity in all epidemiology program areas, representing a substantial increase in estimated need from previous assessments. Overall, current epidemiology staffing translates into 0.72 epidemiologists per 100,000 population, which is lower than the previously recommended ratio of one epidemiologist per 100,000 population. ${ }^{5}$ However, the difference in this ratio between large and small states suggests a more useful approach might entail developing recommendations for staffing adjusted for state population; one epidemiologist per 100,000 population for states of $\geq 5$ million population and approximately 2.5 epidemiologists per 100,000 population for states with populations $<5$ million. The difference in need may result from variation in the way state health departments in more populous states-which typically have well-developed local health departments-operate, compared with those in less populous states. In the latter, state health departments may be more likely to conduct epidemiologic activities typically performed by local health departments in larger states (e.g., foodborne disease investigations), which can require substantial numbers of epidemiologists. Additionally, more populous states are often home to large city health departments, which may have their own complement of epidemiologists, ${ }^{8}$ potentially requiring fewer epidemiologists in the state health department. Other factors affecting the ideal epidemiologist-to-population ratio for states could include overall local health department capacity, public health system governance, organization, and structure.

The proportion of epidemiologists with a degree, training, or coursework in epidemiology has increased with each successive assessment. Because the number of epidemiologists in state health departments has been decreasing, and no evidence exists of substantial personnel turnover, these epidemiologists can be assumed to be the same workers employed in a smaller, better trained workforce. Improvement in epidemiologist training may reflect the unprecedented growth of MPH programs and online degrees available through a distance-learning format. ${ }^{19}$ Degree programs have been complemented by rapidly expanding opportunities for continuing education offered through programs such as the CDC-funded Centers for Public Health Preparedness. However, these centers have experienced significant funding reductions and were completely defunded in August 2010.

Changes in epidemiology program capacity have varied across the ECAs. In the two most recent assessments, the overwhelming majority of states reported substantial- 
to-full capacity for infectious diseases, but the number doing so decreased. Similarly, BT/ER capacity decreased since the 2006 ECA, undoubtedly reflecting declines in federal preparedness funding. As the two program areas most directly bearing on national preparedness for threats from emerging infectious diseases, such as pandemic influenza, the waning number of employees in $\mathrm{BT} / \mathrm{ER}$ and reduced capacity in both is of particular concern and represents a critical vulnerability in our public health preparedness response capacity.

Of additional concern is the decrease in epidemiology capacity for chronic diseases, which, as the leading causes of death, will only increase as the U.S. population ages. Epidemiology capacity for chronic diseases was reported as substantial-to-full for approximately half of all states, and further erosion of already insufficient capacity could endanger public health's ability to successfully address the major causes of morbidity and mortality in the 21 st century.

Reported program capacity in MCH has steadily risen during the last three assessments, which is encouraging given that $\mathrm{MCH}$ addresses challenges characterized by some of the greatest number of years of potential life lost. However, even with increases, fewer than half of all states reported substantial-to-full MCH capacity in 2009, and there was a decline in the absolute number of epidemiologists working in this area since the previous assessment.

Capacity in environmental health and injury epidemiology, two areas growing in importance as public health increasingly focuses on issues such as climate change and aging, also have steadily risen. However, they remain low overall, with only one-third of states reporting substantial-to-full capacity in both. Additionally, both areas sustained declines in absolute numbers of epidemiologists between assessments. In aggregate, while five-year capacity trends in the eight epidemiology program areas are mixed, with half sustaining decreases since the 2006 assessment and the rest remaining static or increasing, significant gaps remain.

Figure 3. Percentage of state health departments reporting substantial-to-full epidemiology and surveillance capacity and capacity to conduct the four epidemiology-related Essential Services of Public Health, by survey year: epidemiology capacity assessments, 2004, 2006, and 2009

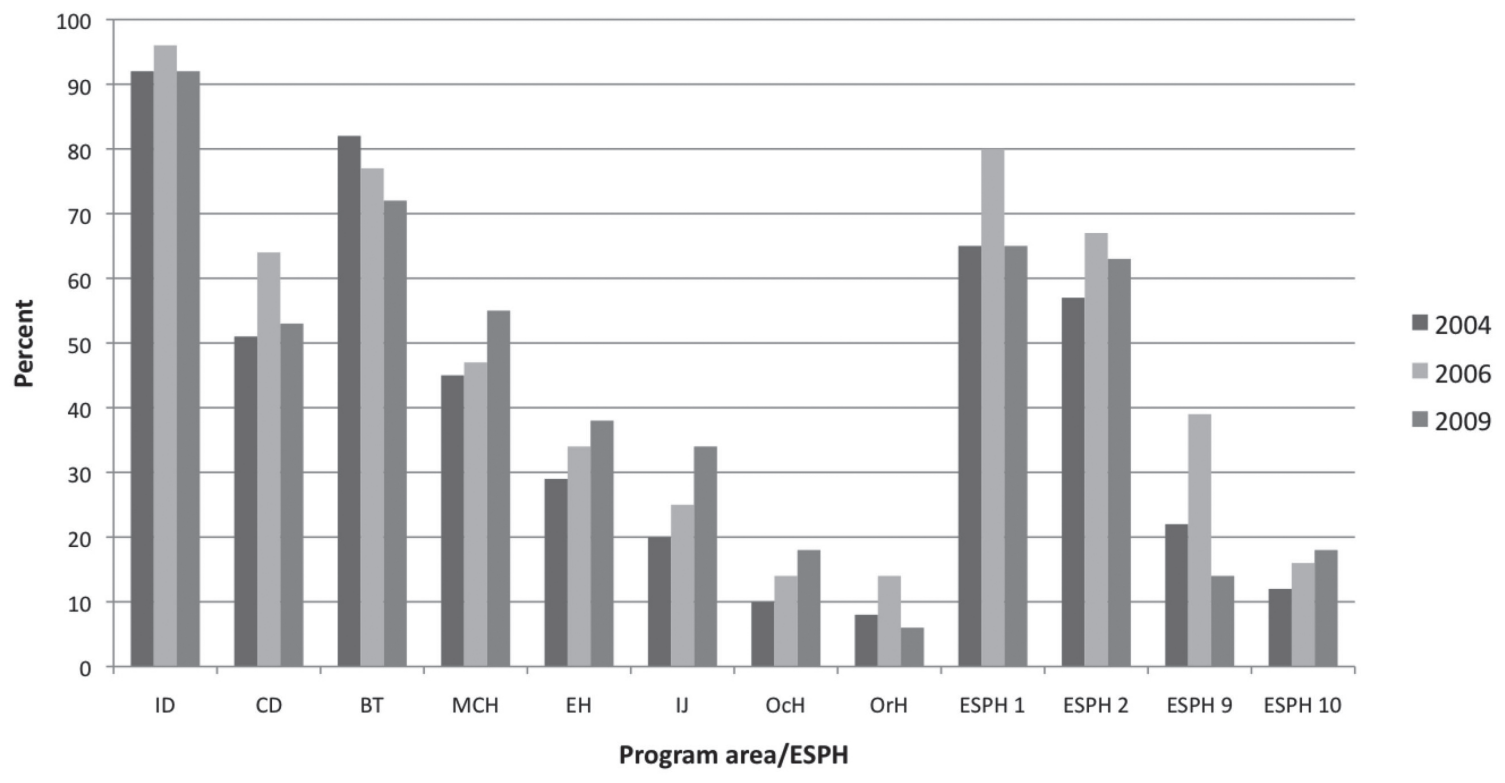

$\mathrm{ESPH}=$ Essential Service of Public Health

$\mathrm{ID}=$ infectious disease

$\mathrm{CD}=$ chronic disease

$\mathrm{BT}=$ bioterrorism

$\mathrm{MCH}=$ maternal and child health

$\mathrm{EH}=$ environmental health

$\mathrm{IJ}=$ injury

$\mathrm{OcH}=$ occupational health

$\mathrm{OrH}=$ oral health

Public Health Reports / January-February 2011 / Volume 126 
The ability of states to provide the four epidemiology-related ESPHs fell after 2006 in three of the ESPHs and increased only minimally in the fourth. A large discrepancy exists between the states' ability to carry out ESPH 1 and 2, for which slightly more than $60 \%$ of states reported substantial-to-full capacity, and their ability to carry out ESPH 9 and 10, for which a consistently low $12 \%-18 \%$ report substantial-to-full capacity. Diminishing capacity to provide the four ESPHs superimposed over low existing capacity for two of the ESPHs reinforces perceptions of a weakening epidemiology capacity in state health departments.

Declining epidemiology infrastructure in state health departments is also reflected by the relatively few states reporting adequate technologic capacity to conduct public health surveillance to manage emerging diseases, such as pandemic influenza. Although federal preparedness funding mandated development of electronic Web-based public health surveillance incorporating laboratory-based reporting in 2001, ${ }^{20}$ most states continue to struggle to initiate Web-based provider or electronic laboratory-based reporting or use of cluster-detection software, greatly hindering their capacity to rapidly detect and respond to infectious disease outbreaks and other public health threats. The lack of technologic capability in state health departments supports the case that overall epidemiology infrastructure in terms of number of personnel and functional program capacity is inadequate and growing more fragile as federal investment falls. Currently, state-level epidemiology capacity is supported through a number of unrelated federal categorical cooperative agreements, with capacity threatened any time funding for one of them decreases. This model needs to be revisited, as epidemiology capacity may best be secured, and strengthened, through a more direct, consolidated funding mechanism combined with greater flexibility in use of categorical emergency preparedness funds.

The federal-state funding ratio for states' epidemiology programs remained largely unchanged from 2006 to 2009. Given the drop in the number of epidemiologists during that same time, it suggests that the decrease in federal funding for epidemiology has been matched, and perhaps even exceeded, by a proportional drop in state funding for these same programs.

\section{Limitations}

Our study was subject to several limitations. For one, the methods used by states to self-assess program capacity and the ability to address the ESPHs may have varied, although CSTE provided worksheets with the ECA to minimize variability and standardize methodology. Secondly, epidemiologists enumerated may have been misclassified, resulting in under- or over-counting; however, states were instructed to adhere to the standard definition of an epidemiologist provided, and individual-level training and educational information was collected for almost three-quarters of epidemiologists. Thirdly, epidemiologists completing individual worksheets may have differed from those not responding.

Fourth, ANOVA testing revealed statistically significant differences in the number of chronic disease epidemiologists between 2004 and 2009. Therefore, reporting states may not accurately reflect chronic disease capacity for all state health departments, although no differences were observed for any of the other program areas in the 2004 or 2009 assessment. Additionally, epidemiologists may have misreported the program-specific level of effort, given the potential overlap in job tasks among BT/ER, infectious diseases, and environmental health. Finally, this assessment only measured epidemiology capacity at the state level and did not encompass local health departments, some of which have considerable epidemiologic capacity.

\section{CONCLUSIONS}

This study presents the most recent findings in a series of national surveys of epidemiology workforce capacity in state health departments. Ongoing measurement of the size and composition of the public health workforce needs to be a national priority, and federal resources should be committed to completing the next round of the national public health enumeration, last conducted in 2000 by the Health Resources and Services Administration. ${ }^{21}$ In parallel with this effort, the U.S. Bureau of Labor Statistics should consider expanding the number and types of public health workforce classifications, ${ }^{22}$ as it does with the physician workforce, to permit a more accurate assessment of people working in public health. CDC recently funded two national Centers of Excellence in Public Health Workforce Research; continued support of these efforts is essential to establish a critical threshold of interest and opportunity for researchers to pursue studies addressing gaps in our understanding of the U.S. public health workforce.

The authors thank Pat McConnon, Jennifer Lemmings, and LaKesha Robinson of the Council of State and Territorial Epidemiologists (CSTE) for making the data available for this study; and Ed Chao of the CSTE for assisting with elements of the data analysis and interpretation.

This article was supported by Cooperative Agreement \#1U38HM000414 from the Centers for Disease Control and Prevention (CDC). Its contents are solely the responsibility of 
the authors and do not necessarily represent the official views of CDC. Additional analysis was completed by the University of Michigan Center of Excellence in Public Health Workforce Studies with support from CDC through a cooperative agreement with the Public Health Foundation. Dr. Boulton has previously served, and Dr. Hadler currently serves, as a paid workforce consultant to the CSTE.

\section{REFERENCES}

1. Association of State and Territorial Health Officials. State public health employee worker shortage report: a civil service recruitment and retention crisis. Washington: ASTHO; 2004.

2. Association of State and Territorial Health Officials. State public health employee worker shortage report. Washington: ASTHO; 2004.

3. Gebbie KM, Turnock BJ. The public health workforce, 2006: new challenges. Health Aff (Millwood) 2006;25:923-33.

4. Baker EL, Potter MA, Jones DL, Mercer SL, Cioffi JP, Green LW, et al. The public health infrastructure and our nation's health. Annu Rev Public Health 2005;26:303-18.

5. Boulton ML, Lemmings J, Beck AJ. Assessment of epidemiology capacity in state health departments, 2001-2006. J Public Health Manag Pract 2009; 15:328-36.

6. Census Bureau (US). Federal, state, and local government employment and payroll data [cited 2009 Jan 4]. Available from: URL: http://www.census.gov/govs/apes/historical_data_2007.html

7. Turnock BJ. Public health workforce trends since 1980: myth or reality? J Public Health Manag Pract 2009;15:105-8.

8. National Association of County and City Health Officials. 2008 national profile of local health departments. Washington: NACCHO; 2009.

9. Department of Health and Human Services (US). Healthy People 2010, volume II: with understanding and improving health and objectives for improving health. 2nd ed. Washington: U.S. Government Printing Office; November 2000. Also available from: URL: http:/ / www.healthypeople.gov/Document/tableofcontents. htm\#Volume2 [cited 2009 Nov 11].
10. Assessment of the epidemiologic capacity in state and territorial health departments-United States, 2001. MMWR Morb Mortal Wkly Rep 2003;52(43):1049-51.

11. Assessment of epidemiologic capacity in state and territorial health departments-United States, 2004. MMWR Morb Mortal Wkly Rep 2005;54(18):457-9.

12. Assessment of epidemiology capacity in state health departments-United States, 2009. MMWR Morb Mortal Wkly Rep 2009;58(49):1373-7.

13. Council of State and Territorial Epidemiologists. 2009 national assessment of epidemiology capacity: findings and recommendations. Atlanta: CSTE; 2009. Also available from: http://www.cste .org/dnn/LinkClick.aspx?fileticket $=\% 2 \mathrm{bS} \%$ 2fEifgcbmM $\% 3 \mathrm{~d} \&$ tabid=36\&mid=1496 [cited 2009 Dec 22].

14. Last JM, editor. A dictionary of epidemiology. 4th ed. New York: Oxford University Press; 2001.

15. Centers for Disease Control and Prevention (US). 10 essential public health services. Atlanta: Council of State and Territorial Epidemiologists; 2008. Also available from: URL: http://www.cdc.gov/od/ ocphp/nphpsp/essentialphservices.htm [cited 2009 Jan 4].

16. SAS Institute, Inc. SAS ${ }^{\circledR}$ : Version 8.0. Cary (NC): SAS Institute, Inc.; 2000 .

17. SPSS, Inc. SPSS ${ }^{\oplus}$ : Version 17.0 for Windows. Chicago: SPSS, Inc.; 2008.

18. Gebbie K, Merrill J, Hwang I, Gebbie EN, Gupta M. The public health workforce in the year 2000. J Public Health Manag Pract 2003;9:79-86.

19. Association of Schools of Public Health. Member schools [cited 2009 Dec 22]. Available from: URL: http://www.asph.org/document.cfm?page $=200$

20. Biological and chemical terrorism: strategic plan for preparedness and response: recommendations of the CDC strategic planning workgroup. MMWR Recomm Rep 2000;49(RR-4):1-14.

21. Health Resources and Services Administration (US). The public health workforce enumeration 2000. Washington: Department of Health and Human Services (US); 2000.

22. Gebbie K, Merrill J, Sanders L, Gebbie EN, Chen DW. Public health workforce enumeration: beware the "quick fix." J Public Health Manag Pract 2007;13:72-9. 